\title{
A specialist herbivore suppresses host plant resistance by inducing a leucine-rich repeat receptor-like kinase
}

\author{
Meng $\mathrm{Ye}^{1}$, Peng Kuai ${ }^{1}$, Lingfei $\mathrm{Hu}^{1}$, Miaofeng $\mathrm{Ye}^{1}$, Hao $\mathrm{Sun}^{1}$, Matthias Erb ${ }^{2}$, and Yonggen \\ $\mathrm{Lou}^{1}$
}

\author{
${ }^{1}$ Zhejiang University - Zijingang Campus \\ ${ }^{2}$ University of Bern
}

April 28, 2020

\begin{abstract}
The mechanisms by which herbivores induce plant defenses are well studied. However, how specialized herbivores suppress plant resistance is still poorly understood. Here, we discovered a rice (Oryza sativa) leucine-rich repeat receptor-like kinase, OsLRRRLK2, which is induced upon attack by gravid females of a specialist piercing-sucking herbivore, the brown planthopper (BPH, Nilaparvata lugens). Silencing OsLRR-RLK2 decreases the constitutive activity of mitogen-activated protein kinase (OsMPK6) and alters BPH-induced transcript levels of several defense-related WRKY transcription factors. Moreover, silencing OsLRRRLK2 reduces BPH-induction of jasmonic acid and ethylene but promotes the biosynthesis of both elicited salicylic acid and $\mathrm{H} 2 \mathrm{O} 2$; silencing also enhances the production of volatiles emitted from rice plants infested with gravid BPH females. These changes decrease BPH preference and performance in the glasshouse and the field. Our study identifies OsLRR-RLK2 as a major susceptibility factor of rice against BPH. It is likely to be employed by BPH to suppress host plant defenses for its own benefit via signaling crosstalk and/or changing the plant's defense-related signaling profile.
\end{abstract}

\section{Hosted file}

LRR2-Main \selectlanguage\{ngerman\} text-Lou0408.doc available at https://authorea.com/users/309906/ articles/440763-a-specialist-herbivore-suppresses-host-plant-resistance-by-inducing-a-leucinerich-repeat-receptor-like-kinase 


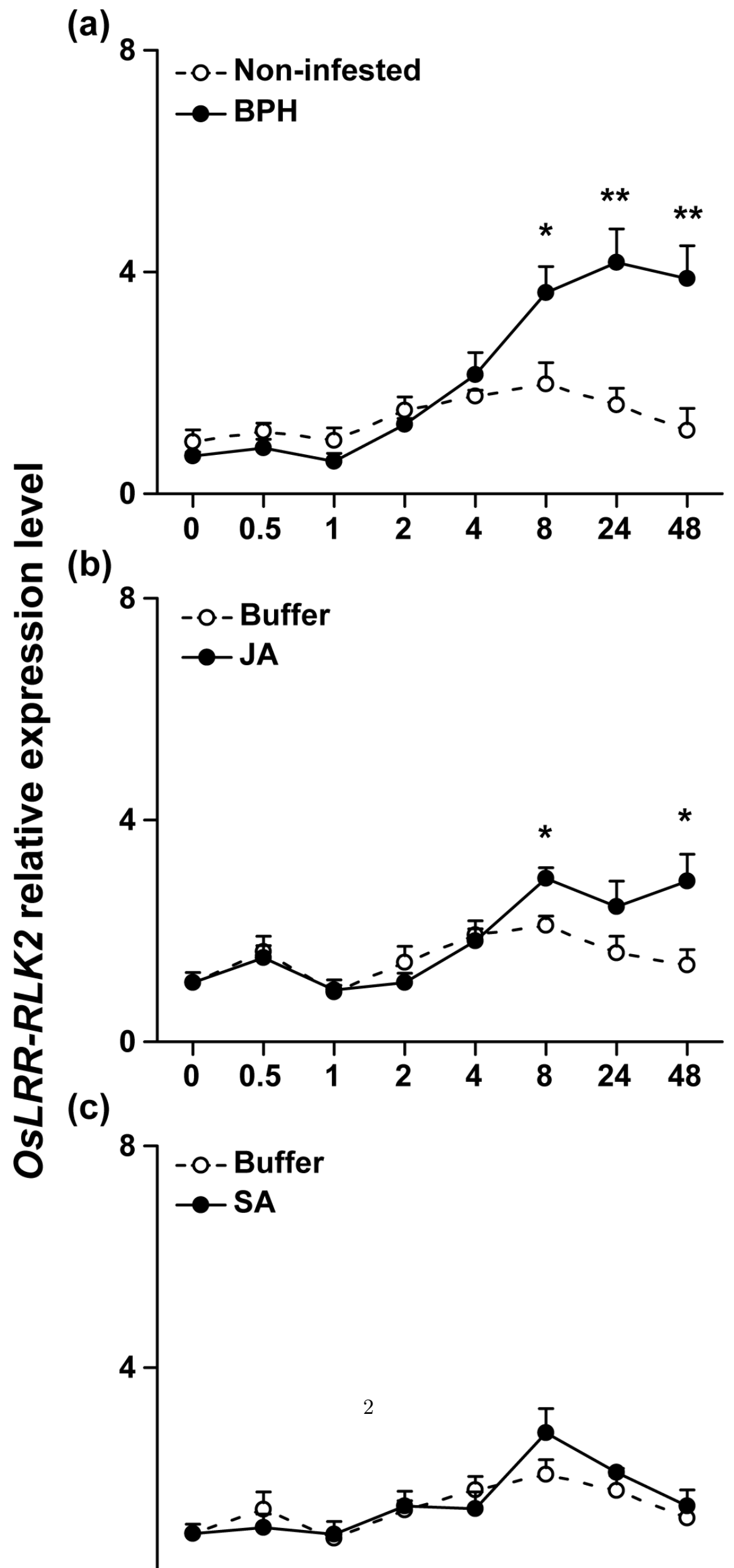


(a)

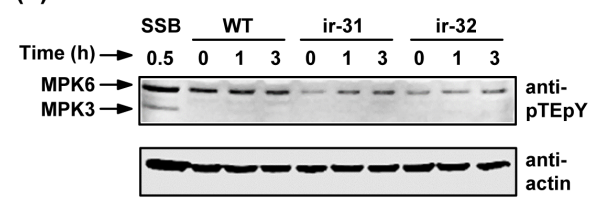

(b)
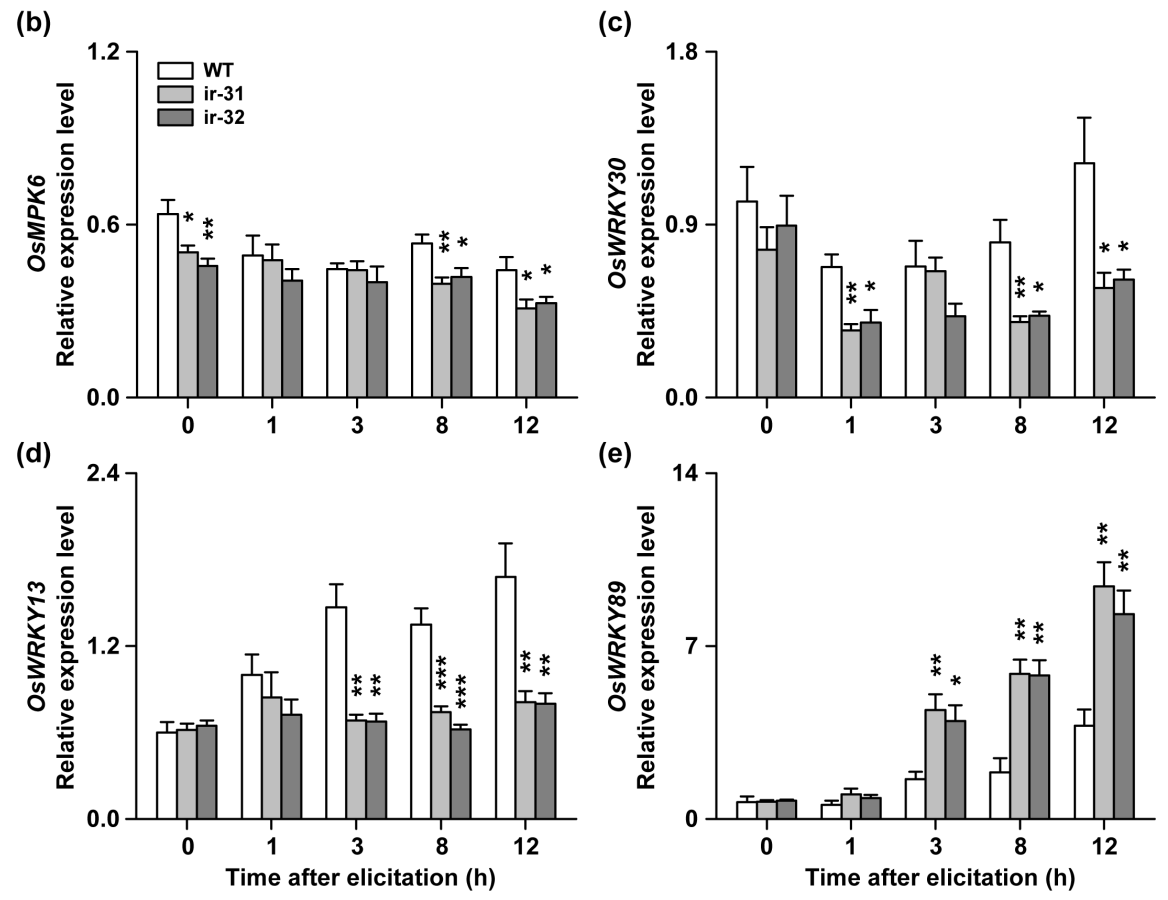

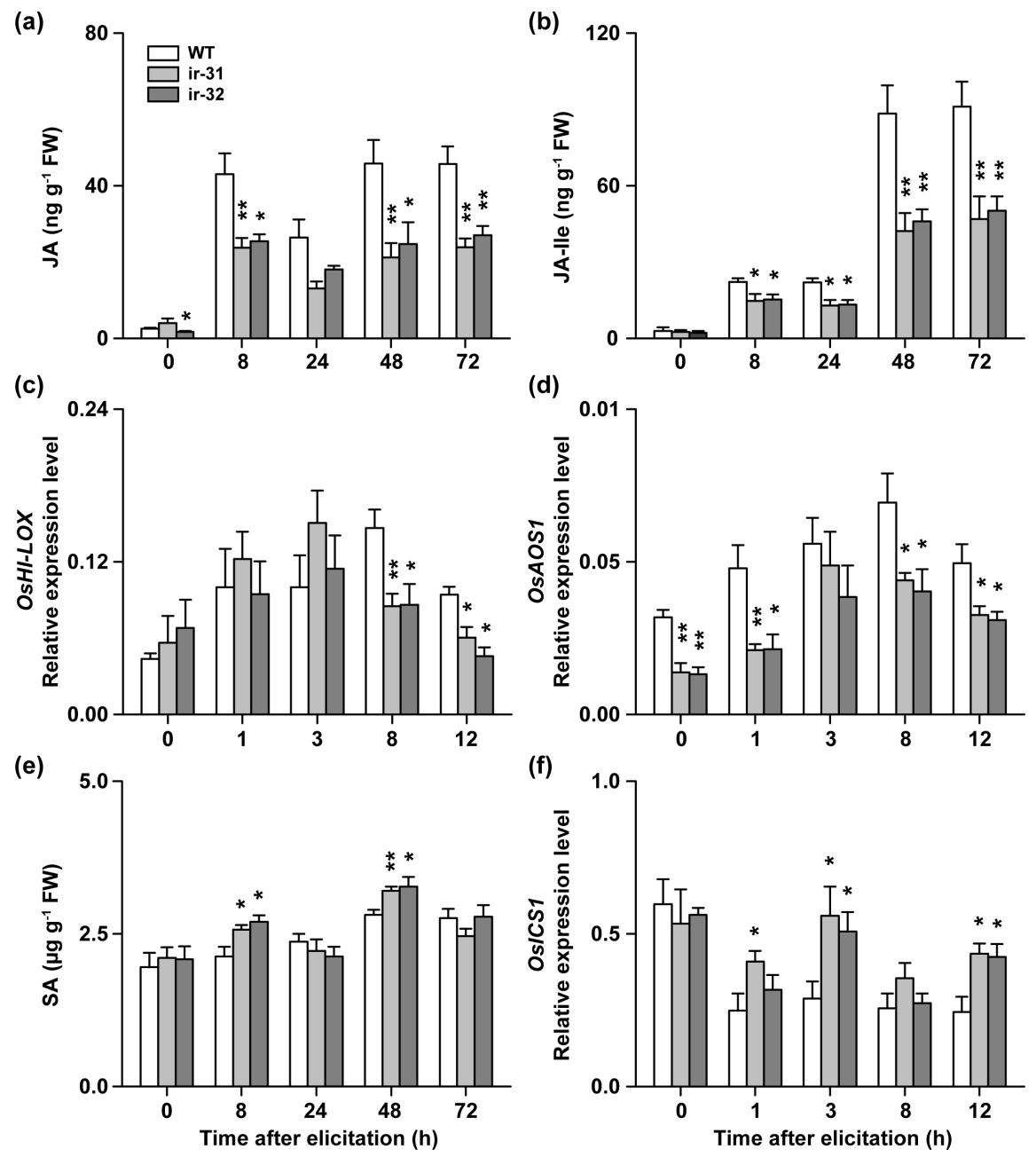

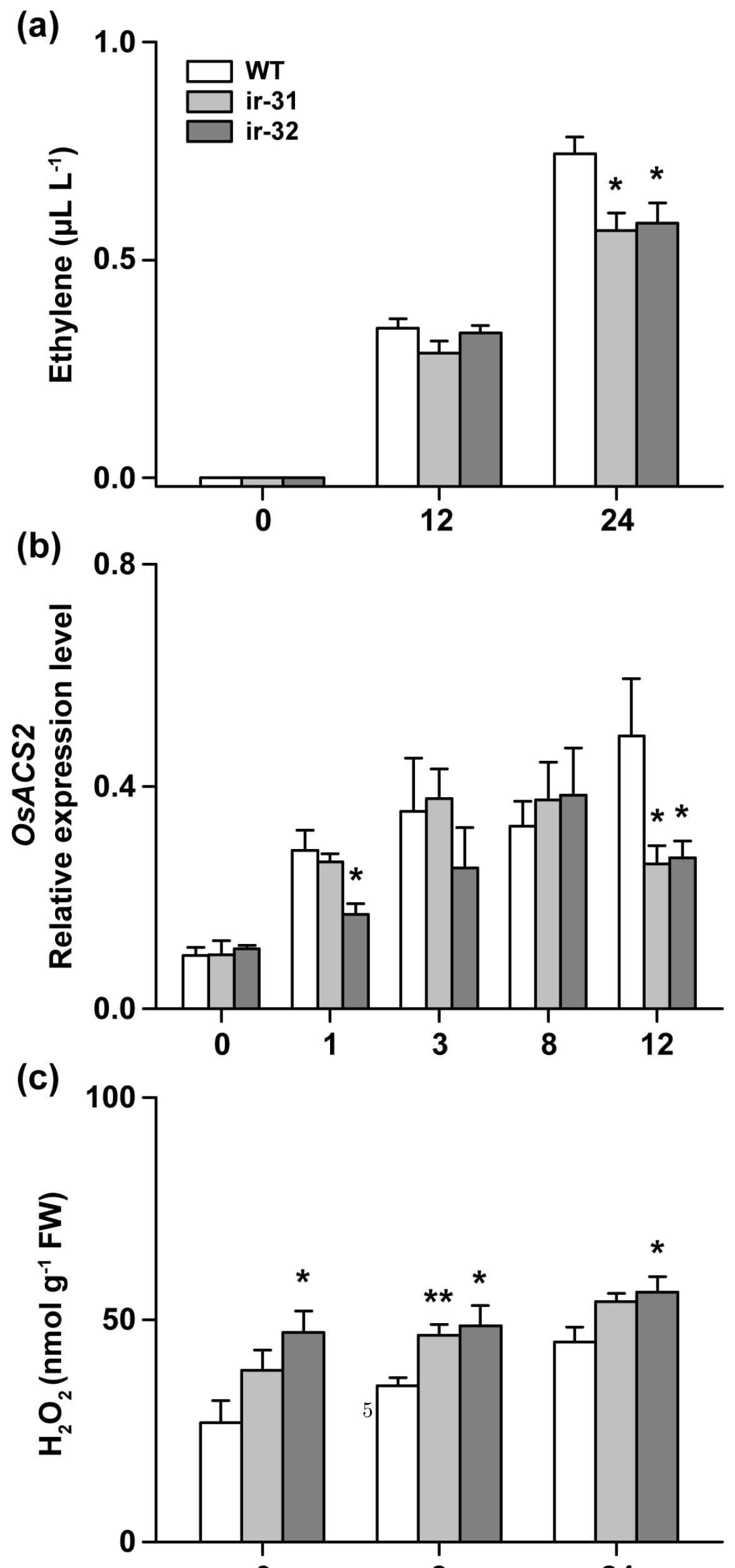

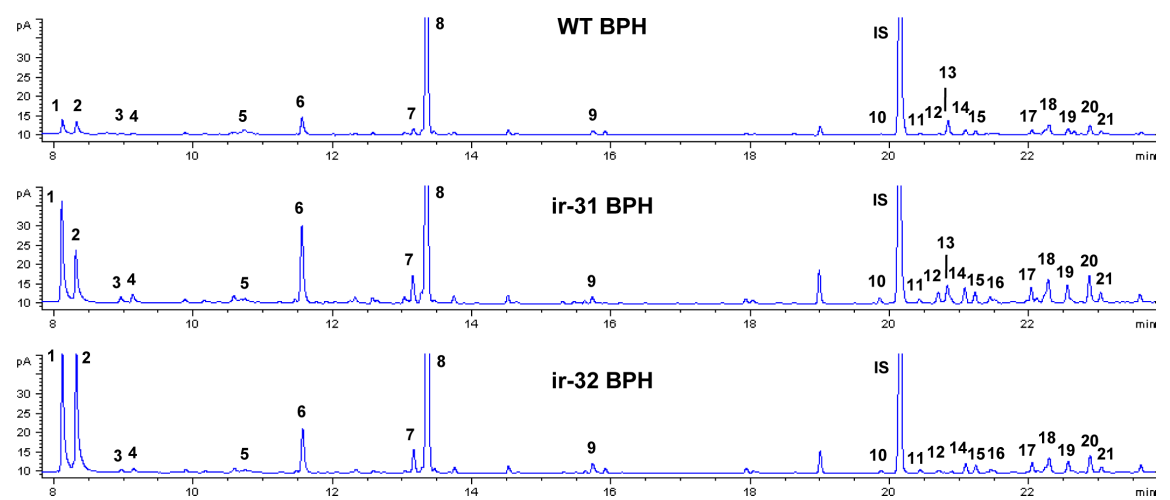

(a)

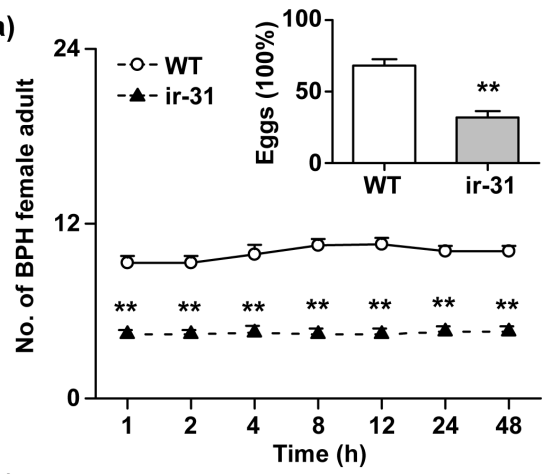

(c)

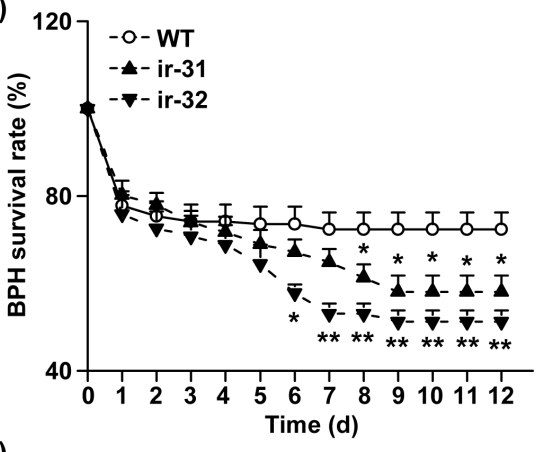

(e)

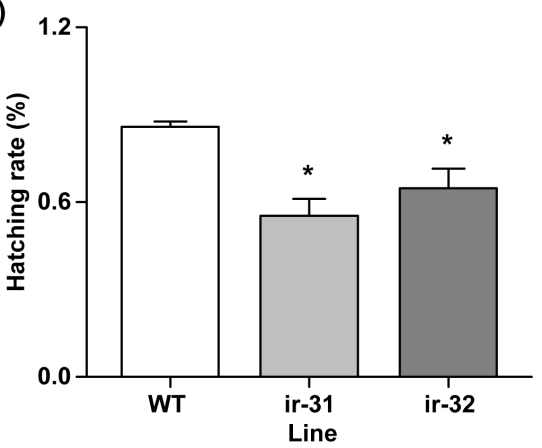

(b)

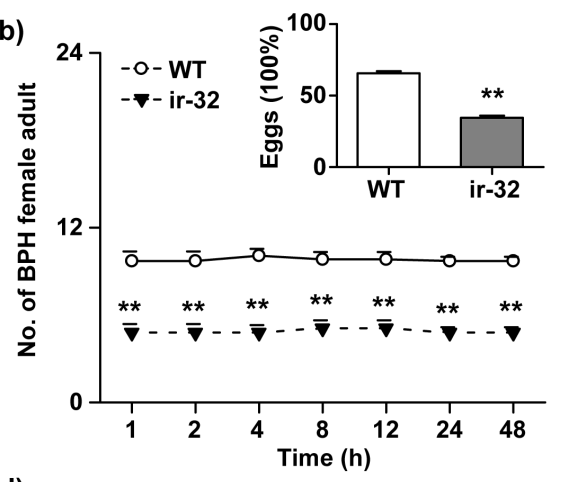

(d)

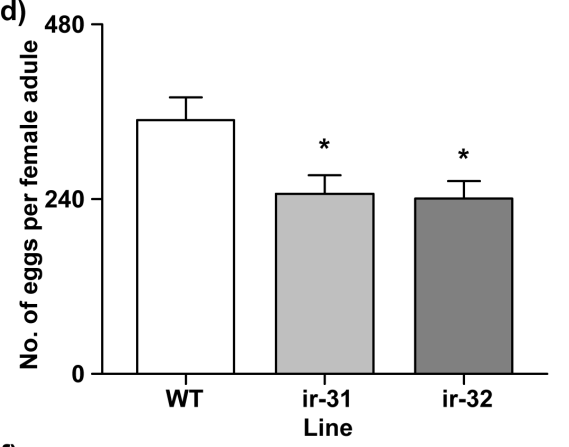

(f)

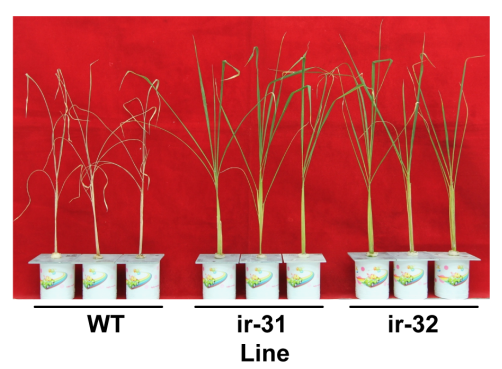



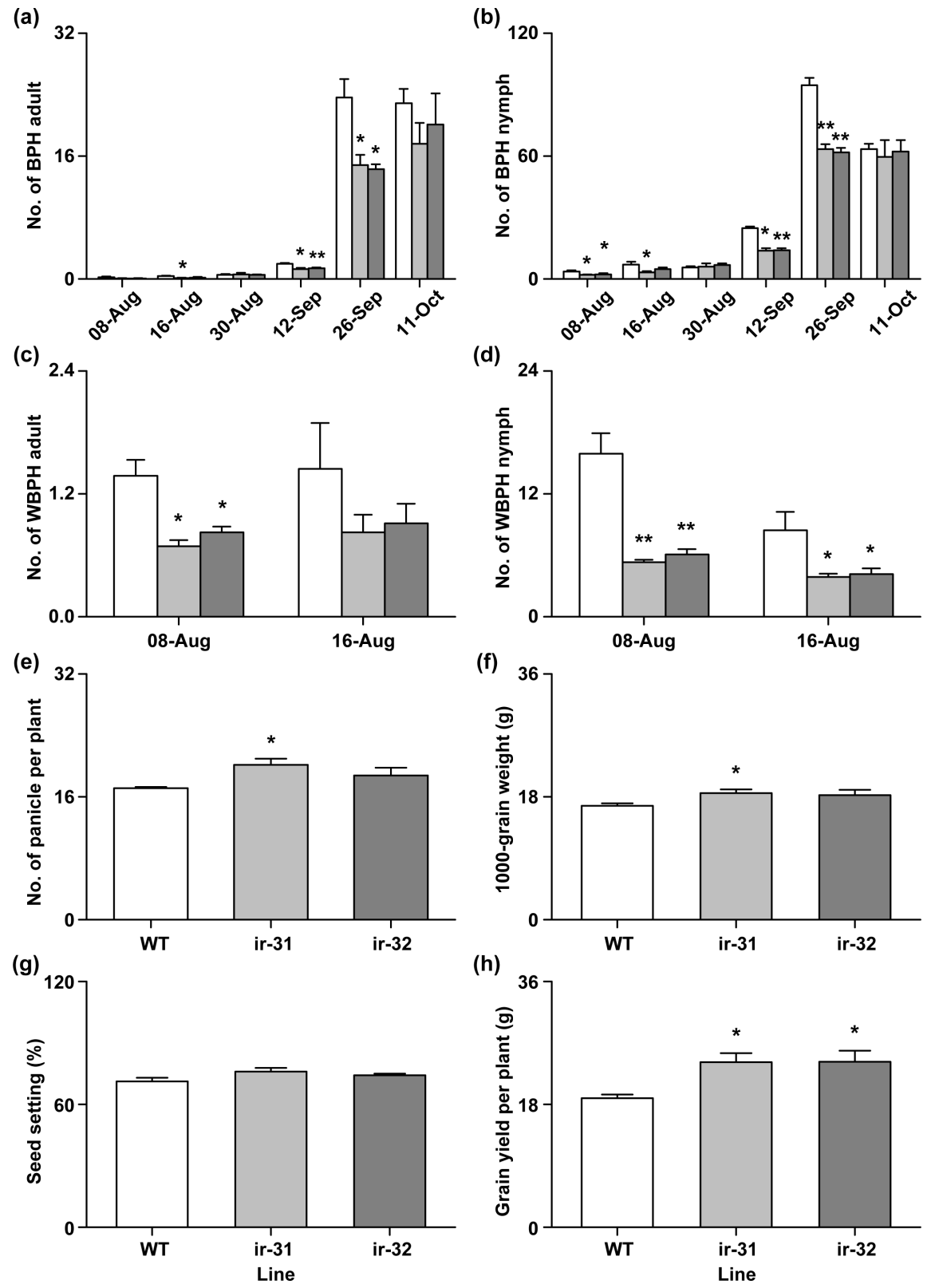